\title{
ANÁLISIS EPIDEMIOLÓGICO DEL CARCINOMA RENAL DE CÉLULAS CLARAS Y CROMOFÍLICO
}

\author{
J.M. GÓMEZ DE VICENTE, L. LLANES GONZÁLEZ, D. SANTOS ARRONTES, \\ V. CHIVA ROBLES, I. ROMERO CAGIGAL, E. REDONDO GONZÁLEZ, \\ A. BERENGUER SÁNCHEZ
}

Servicio de Urología. Hospital Universitario de Getafe. Madrid.

Actas Urol Esp. 27 (1): 22-25, 2003

\section{RESUMEN}

“ANÁLISIS EPIDEMIOLÓGICO DEL CARCINOMA RENAL DE CÉLULAS CLARAS Y CROMOFÍLICO”

OBJETIVO: Encontrar diferencias en las características epidemiológicas de las dos variedades más frecuentes de cánceres renales: el carcinoma renal de células claras y el cromofílico (papilar).

MATERIAL Y MÉTODOS: Análisis retrospectivo de las características epidemiológicas de los pacientes diagnosticados de carcinoma renal en nuestro hospital entre los años 1991 y 2001. Se buscaron diferencias estadísticamente significativas entre las características de los pacientes (edad, sexo y hábito tabáquico) y de los tumores (tamaño, focalidad, estadio, lateralidad y grado).

RESULTADOS: Se diagnosticaron 66 tumores renales, de los cuales $41(62,1 \%)$ eran de células claras y 9 (13\%) cromofílicos. Únicamente se encontraron diferencias estadísticamente significativas entre ambas subpoblaciones en lo referente al tamaño $(\mathrm{p}<0,05)$, siendo mayor en el caso de los de células claras $(7 \pm 3,92 \mathrm{~cm})$ frente a los cromofílicos $(4,89 \pm 1,96 \mathrm{~cm})$. También se apreció una sensible tendencia de los tumores cromofílicos a encontrarse en estadios más precoces en el momento del diagnóstico.

CONCLUSIONES: Los carcinomas renales de células claras y los cromofílicos son las dos variedades más frecuentes de tumores renales. Los cromofilicos son de menor tamaño y suelen encontrarse en un estadio menos avanzado, aunque esta tendencia no se ha podido demostrar en nuestra serie.

PALABRAS CLAVE: Cáncer renal. Epidemiología.

ABSTRACT

"EPIDEMIOLOGIC ANALYSIS OF THE CLEAR-CELL AND CHROMOPHILIC RENAL CARCINOMA "

OBJECTIVE: Finding differences in the characteristics of the two most frequent variants of renal cancers: the clear-cell renal carcinoma, and the chromophilic one.

MATERIAL AND METHODS: Restrospective analysis of epidemilogical characteristics of patients diagnosed of renal carcinoma in our hospital between 1991 and 2001. Statistical differences were searched between patients ${ }^{\prime}$ characteristics (age, sex, smoking habitus) and tumors' characteristics (size, focality, stage, side and grade).

RESULTS: Sixty six renal tumors were diagnosed, of which 41 (62.1\%) were clear-cell tumors and 9 (13\%) were chromophilic. We only found statistical differences between both subpopulations ${ }^{-}$tumor size $(\mathrm{p}<0,05)$, being greater for clear-cell tumors $(7 \pm 3,92 \mathrm{~cm})$ than for chromophilic ones $(4,89 \pm 1,96 \mathrm{~cm})$. We could also appreciate a bias towards a less advanced stage of the chromophilic type, although not statistically significant.

CONCLUSIONS: Clear-cell renal carcinoma and chromophilic renal carcinoma are the two more frequent variants of renal tumors. The chromophilic type is smaller and is usually found in a less advanced stage, although this bias could not be demonstrated in our series.

KEY WORDS: Renal neoplasms. Epidemiology. 
$\mathrm{E}$ carcinoma renal comprende el $2 \%$ de los tumores en EEUU y se produjeron 12.000 muertes por su causa en $1998^{1}$.

En España, se calcula que la tasa de mortalidad ajustada por edad es de 2,9 varones por 100.000 varones/año y de 1,1 mujeres por 100.000 mujeres/año, según estudios europeos de $1992^{2}$. Sin embargo estadísticas recientes arrojan cifras algo mayores, ascendiendo a 5,1 entre los varones y a 1,72 entre las mujeres en 1998. En ese año, murieron 1114 varones y 510 mujeres por cáncer renal (www.isciii.es).

Las dos variedades más frecuentes de carcinoma de células renales (CCR) es el de células claras $(75-85 \%)$ y el cromofílico o papilar $(14 \%)^{3}$.

Recientes estudios han demostrado que se trata de tumores con características citogenéticas diferentes y por lo tanto se puede esperar un comportamiento diferente entre ambas poblaciones ${ }^{4}$.

Nuestro objetivo es estudiar las características epidemiológicas de dichas poblaciones en nuestra serie de tumores renales.

\section{MATERIAL Y MÉTODOS}

Entre los años 1991 y 2001 se diagnosticaron y trataron 67 tumores renales en el Hospital Universitario de Getafe.

El área sanitaria 10 de la Comunidad Autónoma de Madrid comprende una población de 262.843 habitantes (131.380 varones, 131.463 mujeres) según datos del Instituto Nacional de Estadística a 1/1/2001 (www.ine.es).

Se realizó un estudio retrospectivo, valorando las características de los pacientes (edad, sexo, habito tabáquico) y las características de los tumores (tamaño tumoral, focalidad, grado citológico, lateralidad, estadio TNM y Robson).

Empleamos el programa estadístico SPSS para Windows (versión 8.0.0). La edad y el tamaño de los tumores se compararon mediante la $\mathrm{t}$ de Student y el resto de los factores mediante análisis de la $\chi 2$ aplicando el test exacto de Fisher en los casos en los que las frecuencias esperadas eran inferiores a 5 .

\section{RESULTADOS}

De los 67 tumores, 1 fue un oncocitoma que no se tuvo en cuenta a la hora del análisis estadístico. De los 66 tumores restantes, 41 (61\%) fueron de células claras, 9 (13\%) cromofílicos, 5 (8\%) cromófobos, 3 (5\%) sarcomatosos, 1 (2\%) mixto, en 4 (6\%) el patólogo describió la lesión aunque no lo encuadró en ninguna de las variantes conocidas y en 3 casos (5\%) desconocemos el diagnóstico anatomopatológico (Fig. 1).

La incidencia anual ha ido aumentando paulatinamente a lo largo de los años (Fig. 2), llegando a un máximo de 12 casos en el año 2000.

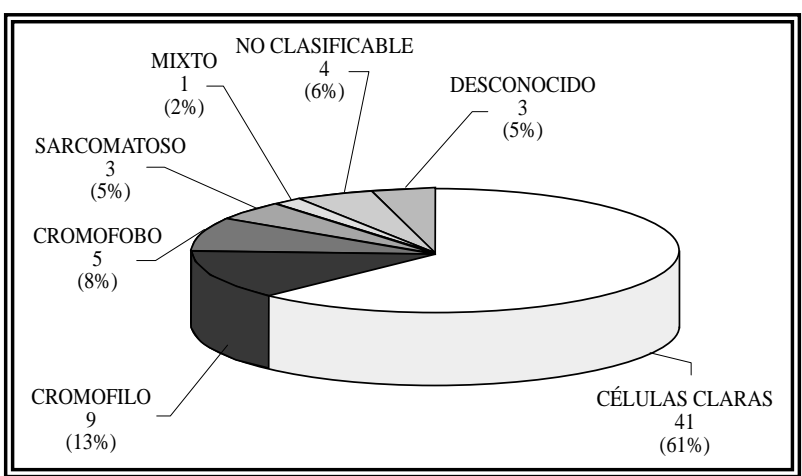

FIGURA 1. Distribución de los 66 tumores renales de nuestra serie.

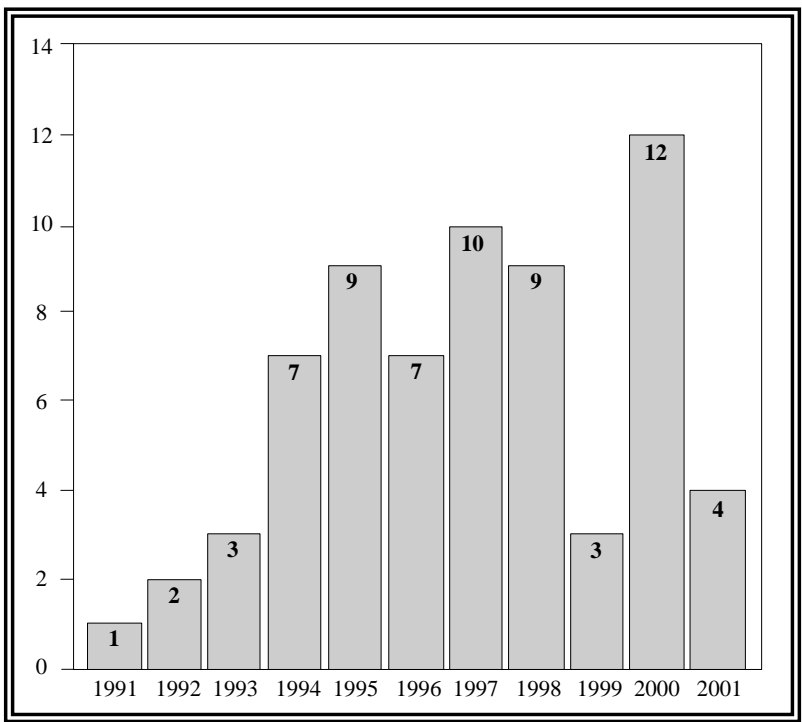

FIGURA 2. Incidencia anual de carcinoma renal en el Hospital Universitario de Getafe.

Los resultados definitivos se muestran en la Tabla I. La incidencia global en nuestra serie fue de 2,51 por 100.000 habitantes. La edad media al diagnóstico de los carcinomas renales de células claras fue de $59 \pm 16,2$ años y de 49,2 \pm 5 en el caso de los cromofílicos, no existiendo diferencias estadísticamente significativas. Tampoco encon- 
CARACTERÍSTICAS DE LA POBLACIÓN

\begin{tabular}{|c|c|c|c|c|}
\hline & & $\begin{array}{c}\text { CCR Células } \\
\text { Claras } \\
\text { (media/ DE) }\end{array}$ & $\begin{array}{c}\text { CCR } \\
\text { cromofílico }\end{array}$ & $\begin{array}{l}\text { Significación } \\
\text { estadística }\end{array}$ \\
\hline \multicolumn{2}{|r|}{ Edad } & $56,7 / 16,2$ & $49,2 / 14,5$ & ns \\
\hline \multirow[t]{2}{*}{ Sexo } & Varón & 22 & 6 & \multirow{2}{*}{$\mathrm{ns}$} \\
\hline & Mujer & 19 & 3 & \\
\hline \multirow[t]{2}{*}{ Fumador } & $\mathbf{S i}$ & 13 & 3 & \multirow{2}{*}{ ns } \\
\hline & No & 28 & 6 & \\
\hline Tamaño & & $7 / 3,9$ & $4,8 / 1,9$ & $p=0,02$ \\
\hline \multirow[t]{2}{*}{ Focos } & Uni & 36 & 8 & \multirow{2}{*}{ ns } \\
\hline & Multi & 5 & 1 & \\
\hline \multirow{2}{*}{$\mathbf{T}$} & $\begin{array}{l}\text { Organoconfinado } \\
\text { (T1/T2) }\end{array}$ & $\begin{array}{c}23 \\
(14 / 9)\end{array}$ & $\begin{array}{c}8 \\
(7 / 1)\end{array}$ & \multirow{2}{*}{ ns } \\
\hline & $\begin{array}{c}\text { No organoconfinado } \\
\text { (T3/ T4) }\end{array}$ & $\begin{array}{c}16 \\
(16 / 0)\end{array}$ & $\begin{array}{c}1 \\
(1 / 0)\end{array}$ & \\
\hline \multirow[t]{2}{*}{$\mathbf{N}$} & NO & 31 & 6 & \multirow{2}{*}{ ns } \\
\hline & N1/ N2 & 1 & 0 & \\
\hline \multirow[t]{2}{*}{ M } & MO & 39 & 9 & \multirow{2}{*}{ ns } \\
\hline & M1 & 2 & 0 & \\
\hline \multirow{6}{*}{ Robson } & I & 23 & 8 & \multirow{6}{*}{ ns } \\
\hline & II & 8 & 0 & \\
\hline & IIIA & 6 & 0 & \\
\hline & IIIB & 1 & 0 & \\
\hline & IIIC & 0 & 1 & \\
\hline & IV & 2 & 0 & \\
\hline \multirow{3}{*}{ Lateralidad } & Derecho & 22 & 6 & \multirow{3}{*}{ ns } \\
\hline & Izquierdo & 16 & 3 & \\
\hline & Bilateral & 3 & 0 & \\
\hline \multirow{3}{*}{$\begin{array}{l}\text { Grados de } \\
\text { Furhman }\end{array}$} & 1 & 9 & 0 & \multirow{3}{*}{ ns } \\
\hline & $2+3$ & $20(11+9)$ & $8(3+5)$ & \\
\hline & 4 & 4 & 0 & \\
\hline
\end{tabular}

tramos diferencias en lo que se refiere al sexo. La proporción de hombres:mujeres fue de 1,15:1 en el caso de los carcinomas de células claras y de 2:1 en los cromofílicos.

El tabaco es un factor de riesgo conocido para el desarrollo del carcinoma renal ${ }^{5}$. Sin embargo no parece que su influencia sea diferente para uno $\mathrm{u}$ otro subtipo celular como demostraron los resultados.
El tamaño fue la única variable que presentó diferencias estadísticamente significativas, encontrando un tamaño medio de $7 \pm 3,92 \mathrm{~cm}$ en los carcinomas de células claras frente a los 4,89 $\pm 1,96 \mathrm{~cm}$ de los cromofílicos ( $\mathrm{p}=0,02$ ). Esto explica que entre los carcinomas cromofílicos confinados al riñón existan $7 \mathrm{~T} 1$ por cada T2, mientras que en el caso de los carcinomas de células claras existen 1,5 $\mathrm{T} 1$ por cada $\mathrm{T} 2$. 
No encontramos diferencias en cuanto a la presencia de adenopatías o metástasis al diagnóstico. Aplicando el estadiaje de Robson tampoco encontramos diferencias estadísticamente significativas, aunque la práctica totalidad de los carcinomas renales cromofilicos fueron órganoconfinados.

En algunos de los informes anatomopatológicos no se informó sobre el grado nuclear de Furham y en esos casos no se atribuyó grado citológico al tumor, y por lo tanto no se tuvo en cuenta a la hora de realizar el análisis estadístico. Agrupamos los grados 2 y 3 basándonos en el artículo original de Furham ${ }^{6}$ donde no se encontraron diferencias de supervivencia entre estos dos grupos. En este caso tampoco encontramos diferencias entre ambas subpoblaciones.

Finalmente se compararon la lateralidad y la focalidad (uni o multifocal), no encontrando tampoco diferencias en estos casos.

\section{DISCUSIÓN}

Los CCR de células claras se caracterizan por presentar una delección puntual en una o ambas copias del cromosoma 3p, mientras que los CCR cromofilicos presentan alteraciones cromosómicas completas como monosomía $\mathrm{Y}$, trisomía 7 y $17^{3,4,7}$. Aunque ambos tumores proceden de células del tubo contorneado proximal, se trata de tumores con distinto pronóstico ${ }^{8,9}$. Por ello hemos intentado buscar diferencias en las características epidemiológicas de ambas poblaciones.

Aunque nuestra serie de tumores renales no es muy numerosa, nos permite sacar algunas conclusiones. La proporción de carcinomas renales de células claras y cromofílicos es similar a la de otras series descritas en la literatura ${ }^{3}$. No obstante, los CCR cromofílicos suelen encontrarse en un estadio local poco evolucionado. En una serie de 224 CCR, donde 34 eran tumores papilares, el 85,3\% se encontraban en un estadio I de Robson ${ }^{8}$. En nuestra serie, el $88,8 \%$ se encontraban en un estadio I, y tan sólo 1 de los 9 tumores se encontraba en un estadio más avanzado.

Se ha descrito un menor tamaño de los tumores cromofilicos, así como un mejor pronóstico frente a los carcinomas renales de células claras ${ }^{9}$. En nuestro medio el tamaño medio de los tumores cromofílicos fue de $4,8 \mathrm{~cm}$ (mediana $4 \mathrm{~cm}$ ) frente a los $7 \mathrm{~cm}$ (mediana $6 \mathrm{~cm}$ ) de los de células claras.
A pesar de un menor tamaño y un estadio más precoz de los tumores cromofílicos, la mayoría $(62,5 \%)$ eran de un grado citológico elevado. Esto puede ser explicado por la baja reproducibilidad y pobre concordancia interobservador de los sistemas de gradación de los tumores renales, incluido el de Furham ${ }^{10}$.

A diferencia de otros estudios en los que era habitual la multifocalidad del carcinoma renal cromofílico $^{8}$, en nuestra serie, tan sólo 1 de los 8 tumores era multifocal.

\section{CONCLUSIONES}

Los CCR cromofílicos y de células claras son los tumores malignos más frecuentes en el riñón. Los CCR cromofílicos suelen ser de menor tamaño y tienden a encontrarse en un estadio menos avanzado. No parecen existir diferencias etarias o sexuales entre ambas poblaciones.

\section{REFERENCIAS}

1. TOVANI A, LA VECCHIA C: Epidemiology of renal cell carcinoma. J Nephrol 1997; 10: 93-106.

2. LA VECCHIA C, LERI F, LUCCHINI F, NEGRI E: Descriptive epidemiology of kidney cancer in Europe. J Nephrol 1992; 5: $37-43$.

3. MOTZER RJ, BAUDER NH, NANUS DM: Renal-cell carcinoma. NEJM sept 19, 1996; 335: 865-875.

4. KOVAKS G, FUZESI L, EMANUAL A, KUNG HF: Cytogenetics of papillary renal cell tumors. Genes Chromosomes Cancer 1991; 3: 249-255.

5. YU MC, MACK TM, HANISCH R, CICIONI C, HENDERSON $\mathrm{BE}$ : Cigarette smoking, obesity, diuretic use and coffee consumption as risk factors for renal cell carcinoma. J Natl Cancer Inst 1986; 77: 351-356.

6. FURHAM SA, LASKY LC, LIMAS C: Prognostic significance of morphologic parameters en renal cell carcinoma. Am J Surg Pathol 1982; 6: 655.

7. PRESTI JC JR, RAO PH, CHEN Q, REUTER VE, LI FP, FAIR WR, JHANWAR SC: Histopathological, cytogenetic and molecular characterization of renal cortical tumors. Cancer Res 1991, 51: 1.544-1.552.

8. MANCILLA-JIMENEZ R, STANLEY RJ, BLATH RA: Papillary renal cell carcinoma: a clinical, radiologic, and pathologic study of 34 cases. Cancer 1976; 38: 2.469-2.480.

9. SENE AP, HUNT L, MCMAHON RF, CARROLL RN: Renal carcinoma in patients undergoing nephrectomy: analysis of survival and prognostic factors. Br J Urol 1992; 70: 125-134.

10. LANIGAN D, CONROY R, BARRY-WALSH C, LOFTUS B, ROYSTON D, LEADER M: A comparative analysis of grading systems in renal adenocarcinoma. Histopathology 1994; 24: 473-476.

Dr. J.M. Gómez de Vicente

Servicio de Urología. Hospital Univ. de Getafe

Ctra. de Toledo km. 12,500

28905 Getafe (Madrid)

(Trabajo recibido el 10 junio de 2002) 